\title{
PREFAZIONE DEL TRADUTTORE
}

Agli specialisti di Neurologia, Oculistica e Otorinolaringoiatria, cosi come agli studenti di medicina questa seconda parte del volume terzo del Trattato di anatomia patologica speciale del Kaufmann sarà di grande utilità, per la ricchezza delle notizie e della bibliografia come per l'efficacia delle illustrazioni.

I proff. Massimo del Bo per la patologia dell'udito e Demetrio Spinelli per quella dell'apparato visivo hanno rivisto accuratamente il testo. Il prof. Luigi Matturri ha svolto una collaborazione costante e preziosa nel lavoro di correzione delle bozze e di redazione. Ad essi va il mio più vivo ringraziamento, che desidero estendere alla Casa Editrice Vallardi per la parte tipografica e al sig. Osvaldo Ciarrocca per il lavoro fotografico.

Alfonso Giordano 
